\title{
Rheological Behaviour of Injection Moulded Oil Palm Empty Fruit Bunch Fibre - Polypropylene Composites: Effects of Electron Beam Processing Versus Maleated Polypropylene
}

\begin{abstract}
In this study, oil palm empty fruit bunch fibres (EFB) reinforced polypropylene (PP) composites were prepared by two different methods i.e. physical treatment of the composites using electron beam irradiation and chemical treatment by adding coupling agent such as maleated PP. The composites pellets were then subjected to injection moulding for preparation of test pieces for physical, mechanical and rheology properties study. Haake Rheometer RS 150 was used to study the changes in viscosities of the composites at different shear rates. The melt flows of the composites were also measured to complement and confirm the rheology behaviour of the composites. Upon irradiation, the viscosity of PP decreased due to chain scission and the sudden drop of viscosity at high shear rate was not significant. The presence of EFB fibre in the PP matrix was obviously interrupted the flow ability of PP that cause viscosity to increase. Upon irradiation of $\mathrm{EFB}=\mathrm{PP}$, the viscosity of the composites decreases. This indicate that no crosslinking occur between EFB and PP during melt mixing although PP and EFB active radicals were present in the composites. On the other hand, the additional malaeted PP increases interfacial adhesion that result in enhance resistance to flow, thus significantly increased in the viscosity of the composite. The addition of $6 \%$ maleated PP exhibited significant drop in viscosity of the composites. These results were further confirmed by the melt flow measurement of irradiated $\mathrm{EFB}=\mathrm{PP}$ with reactive additives and maleated PP. The rheological behaviour of the composite is important parameters in plastic processing particularly in relation to the shear rates for injection moulding and extrusion processes.
\end{abstract}

Keyword: crosslinking, electron beam, empty fruit bunch fibre, maleated polypropylene, rheology 